\title{
Large Eddy Simulation of Natural Extraction and Thermal Stratification Effects in Corridor Fires
}

\author{
I. B. Abdellaziz ${ }^{1}$, M. Bouterra ${ }^{1}$, O. Vauquelin ${ }^{2}$ and A. E. Cafsi ${ }^{1}$ \\ ${ }^{1}$ Laboratory of Energetics, Thermal and Mass Transfers (LETTM), University of Tunis El Manar, Faculty of \\ Sciences of Tunis, El Manar II, 2092, Tunis, Tunisia. \\ ${ }^{2}$ Aix-Marseille University, CNRS, IUSTI UMR 7343, 13453 Marseille, France.
}

$\dagger$ Corresponding Author Email: benabdellazizimen@gmail.com

(Received April 14, 2018; accepted July 30, 2018)

\begin{abstract}
The natural smoke extraction in a corridor with a vertical duct is analyzed numerically using the software Fire Dynamics Simulator. A parametric study is conducted to investigate the influence of the duct geometry (i.e. section, shape and height) on the smoke extraction efficiency. Results show that the section of the duct appears as the most influential geometrical parameter. There exists an optimal duct section, for which, the hot smoke adheres to the left and right walls of the duct. In this case, the mixing process and the stack effect are the dominating factors influencing the natural extraction performance. The thermal stratification is also investigated using the Newman parameter to quantify the smoke stratification stability. It was found that this parameter is predicted by the two-dimensional nature of the flow pattern in the downstream direction and that the degree of stratification decreases as the duct section and height increase.
\end{abstract}

Keywords: Tunnel fires; Stratification; Fire dynamics simulator.

\section{INTRODUCTION}

Channel-like structures, such as tunnel and corridor, is a common geometric design. Tunnel fires often cause severe disasters. Such accidents include (Hong, 2004; Carvel and Marlair, 2005; Gann and Babrauskas, 1994), e.g. a subway tunnel fire in Daegu of Korea in February, 2003, killed 198 people, a fire in Mont Blanc tunnel in March,1999, killed 39 people and a corridor fire at the Hillhaven Nursing Home of Norfolk, Virginia in October, 1991, killed 10 people. Statistics have shown that smoke is the most dangerous agent in fires (Alarie, 2002). To extract heat and smoke in a fire in time and ensure personnel safety and tunnel structure, an effective smoke extraction system is primordial. General smoke extract methods in tunnel at present include the mechanical ventilation and the natural ventilation which use natural smoke vents or smoking duct.

Compared with mechanical ventilation that requires a large space of excavation for installation of ventilation equipments along with electrical energy consumption, natural ventilation using vertical ducts does not consume power in the operation for discharging gas.

In tunnel fire research, many studies have conducted research on ventilation performance realization by mechanical exhaust system ( $\mathrm{Ji}$ et al., 2010; Hwang and Edwards, 2005). Vauquelin and Mégret (2002), have investigated experimentally the smoke extraction of a transverse ventilation system. The tunnel is equipped with two ducts located one on either side of a fire source in order to study the influence of their location and their shape on the exhaust system efficiency in a first step. Then the effect of the fire heat release rate will be presented in a second step. When the exhaust flow rate has to be about three times the buoyant flow rate produced by the source, they conclude that the efficiency of $100 \%$ is reached for 10 and $20 \mathrm{MW}$. The effect of forced ventilation on a buoyant fluid released continuously was studied by Vauquelin (2008) in a small-scale tunnel. The originality of the model was to use a mixture of air and helium to experimentally simulate the smoke fire plume. For the longitudinal control, he demonstrated that the effect of the tunnel geometry is an important parameter which must be considered to evaluate the model prediction for the critical velocity. For the transverse control, he claimed that in the case of plug-holing the ventilation system output (VSO) obviously strongly decreases.

There are only a few preliminary studies on the natural ventilation system with vertical duct. The influence of the cross-sectional area and aspect ratio of the duct on natural ventilation in urban road 
tunnel fires by Large Eddy Simulation (LES) were analyzed by Ji et al. (2013). He demonstrated that when the cross-sectional aspect ratio increases to a certain value, the hot smoke will be adhered to the upstream wall of the duct that will be exhausted through the upstream region and this latter is predicted by using the 2D flow pattern interpretation. Using the LES of FDS (Fire Dynamics Simulator) code (version 5) Zhong et al. (2013) analyzed the smoke flow characteristics of a road tunnel fire under the combined function of a longitudinal wind and stack effect of the duct. The effect of the vertical duct height on natural ventilation is investigated experimentally by Ji et al. (2012), two special phenomena, plug-holing and turbulent boundary layer separation, were influenced by the effect of smoke exhaustion.

Delicate quantitative analyses on how parametrical conditions of a vertical duct location in the tunnel roof influence the natural ventilation performance have rarely been addressed. The thermal stratification quantification based on Newman (1984) has also rarely been discussed.

In order to investigate the effects of the duct geometric on the natural smoke exhausting efficiency, a set of numerical simulations are conducted in the present work. The duct efficiency, the temperature distributions in the tunnel and in the duct for different duct geometries are numerically studied to illustrate the influence of the mixing and plug-holing processes on the smoke-air interface, and thus on the natural ventilation performance. The influence of the stratification parameter on the smoke layer stability is also investigated.

\section{NUMERICAL MODELING}

\section{1 Fire scenario Analysis}

The simulation region of the tunnel was $6 \mathrm{~m}$ long, 2 $\mathrm{m}$ wide and $0.9 \mathrm{~m}$ high. A fire source of a heat release rate (HRR) of $20.21 \mathrm{~kW}$ was located $1.2 \mathrm{~m}$ away from the left entrance (Fig. 1(a)). The model tunnel in current research was taken from the following experimental conditions implemented by Ji et al. (2012).

A $0.8 \mathrm{~m}$ high duct is set up $4.2 \mathrm{~m}$ away from the left opening side and is topped vertically in the tunnel ceiling (Fig. 1(b)). Three series of fire-scenarios are simulated to investigate the influences of the duct section, height and shape, respectively. In the first series, the duct height is fixed $0.8 \mathrm{~m}$ and the section is varied from $0.01 \mathrm{~m}^{2}$ to $0.36 \mathrm{~m}^{2}$ (for each case, there is always equality between the length and the width). In the second series, the section is fixed $0.09 \mathrm{~m}^{2}(0.3 \mathrm{~m} \times 0.3 \mathrm{~m})$ and the height is varied from $0.1 \mathrm{~m}$ to $1 \mathrm{~m}$ with an interval of $0.1 \mathrm{~m}$. However, in the third series, the duct height is again $0.8 \mathrm{~m}$ and the aspect-ratio will be changed. The aspect-ratio (r) presents the ratio between the length and the width duct $(r=L / l)$. It is varied from $r=0.0625$ to 16 with an interval of 4- multiplication. So, for several values of $r(r=0.0625,0.25,1,4$ and 16), the duct always features $0.1024 \mathrm{~m}^{2}$. In addition, the fire scenario without the vertical duct is also simulated.
Figure 1(a) shows the geometry of the tunnel used in a reduced scale model. Figure 1(b) illustrates the duct under the ideal condition $(0.3 \mathrm{~m}, 0.3 \mathrm{~m}, 0.8 \mathrm{~m})$, where the upper part is submitted to the "open" structure of the FDS. The temperature is fixed under the ambient condition at $20^{\circ} \mathrm{C}$ and the schematic diagram of the model with FDS simulation is shown in Fig. 1(c).
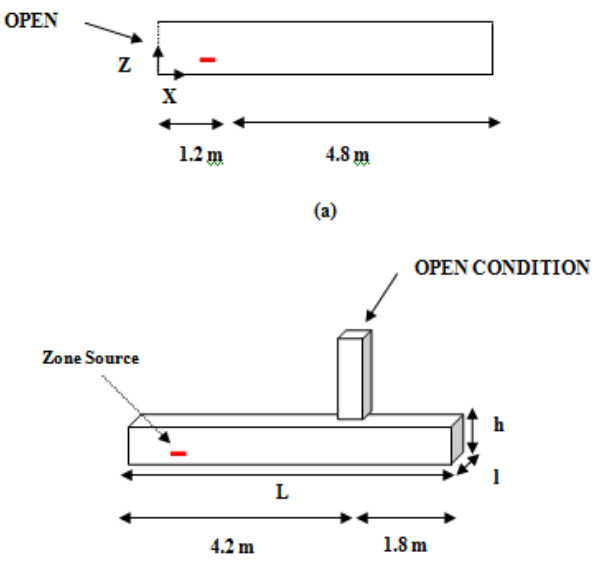

(b)

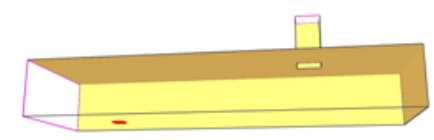

(c)

Fig. 1. Schematic 2D (a) and 3D (b) views of the tunnel, and FDS computational model.

\subsection{Fire Dynamics Simulator}

CFD simulation has now become an important tool for fire science (Mos, 2005). Some CFD solvers have been widely used, such as Fire Dynamics Simulator (FDS) (Mc Grattan et al., 2010) which was developed by the US National Institute of Standards and Technology (NIST). The Fire Dynamics Simulator (Mc Grattan et al., 2010), is a model of fire-driven fluid flow, coded for this work in Fortran input record and run from the command prompt. The model solves numerically the equations of Mass, Momentum and Energy (Mc Grattan et al., 2010) appropriate for low Mach number. Turbulence methods that are commonly used in CFD are based on the Reynolds Averaging Navier-Stokes equation (RANS) method, Large Eddy Simulation (LES) and Direct Numerical simulation (DNS). The LES model, which is widely used in the study of fire-induced smoke flow behavior, is selected here. The Sub-Grid-Model (SGM) commonly used in LES is developed originally by Smagorinsky (1963). According to many validation works, the constants $C s, P r$ and $S c$ are defaulted in FDS as $0.2,0.2$ and 0.5 , respectively. In our study, and in order to set these parameters, we tested many values. The 0.2 for the $C s$ number and 0.5 for the $P r$ number allowed for a fusty convergence of the model. The CourantFriedrichs-Lewy (CFL) criterion is used in FDS for 
justifying numerical convergence. The time step is constrained by the convective and diffusive transport speeds via this condition (Mc Grattan et al., 2010):

$\delta t . \max \left(\frac{\left|U_{i j k}\right|}{\delta X}, \frac{\left|V_{i j k}\right|}{\delta Y}, \frac{\left|W_{i j k}\right|}{\delta Z}\right)<1$

where $U_{i j k}, V_{i j k}$ and $W_{i j k}$ are the components of the velocity vector, and $\delta X, \delta Y$ and $\delta Z$ the dimensions of the smallest grid cell in the $x, y$ and $z$ directions.

\subsection{Validation of Results}

This section is devoted to present the validation of our numerical code. Figure 2 exhibits a comparison of our numerical results obtained using the FDS software, with the experimental results of $\mathrm{Ji}$ et al. (2012) which measured the temperature as a function of time at several positions on a vertical line of tunnel.

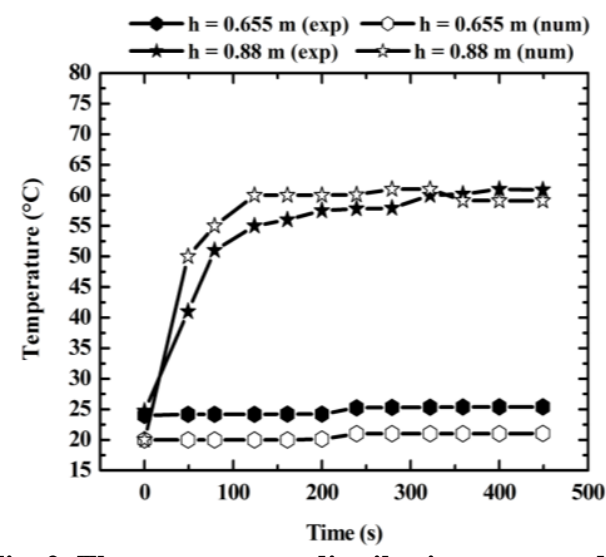

Fig. 2. The temperature distribution measured in the corridor: Curve Validation.

It is very clear that the steady stage is determined as $150-250 \mathrm{~s}$ and the average parameter of temperature calculated by averaging the values within this range. It can be seen that the thermocouple tree (at $h=0.655 \mathrm{~m}$ ) where the temperature rises, are almost approaching to $20-25^{\circ} \mathrm{C}$, which illustrates the whole thermocouple is located in the air layer at the lower part of the tunnel. For example, we can notice that at $250 \mathrm{~s}$, the temperature reaches $25^{\circ} \mathrm{C}$ under the experiment of Ji et al. (2012). At the same time, our numerical result reached $21^{\circ} \mathrm{C}$. It is obvious that in case of thermocouple tree at $h=0.88$, the temperatures rise sharply from the ambient temperature to a relatively steady value at about $150 \mathrm{~s}\left(60^{\circ} \mathrm{C}\right)$. This latter can be explained by the fact that the upper part of the thermocouple is located in the smoke layer at the upper part of the tunnel. For example, we can notice that the temperature achieves at about $57^{\circ} \mathrm{C}$ at $250 \mathrm{~s} \mathrm{(Ji} \mathrm{et}$ al., 2012). However, our numerical results find $60^{\circ} \mathrm{C}$.

It can be found that the values of the temperature for each height ( $h=0.655$ and $0.88 \mathrm{~m}$ ) under the smoke vent without exhausting are in good agreement with experiments performed previously.

\section{EFFICIENCY OF NATURAL DUCT EXTRACTION}

The primary driving force of a vertical ceiling duct in tunnel fire is to discharge smoke and weaken its impact on tunnel structures and permits the evacuation of occupants. When the smoke spreads to the area beneath the duct, it will be exhausted because of the vertical inertial force induced by stack effect. However, under the action of vertical inertia force, the disturbance on smoke air interface will be strengthened greatly, leading to a lot more fresh air mixed into the smoke layer, some of which is discharged through the duct, and the other overflows to the downstream of the tunnel. So, the amount of air which is penetrated into the smoke or drawn directly into the duct from the lower layer due to the plug-holing phenomenon must be very small. Based on this finding, $100 \%$ efficiency will be achieved only by increasing the convective heat release rate (convective HRR) of the smoke extract system and the convective HRR of smoke exhausted by the duct is the key parameter which concerns us most, according to (Vauquelin and Mégret 2002) definitions.

These authors defined the efficiency of an exhaust duct to characterize the transverse ventilation system. It constitutes the ratio of the extracted smoke VFR (Volume Flow Rate) to the produced smoke VFR and can be expressed as follows:

$\varepsilon=\frac{Q_{e s}}{Q_{s}}$

In this study, we will apply this definition to evaluate the performance of natural ventilation with vertical duct. We will be interested in calculating the convective HRR under different transverse planes for several longitudinal displacements along the tunnel:

- The $Q_{(-)}$is the produced HRR calculated in the upstream direction of the source area.

- The $Q_{(+)}$is the produced HRR calculated in the downstream direction of the source area.

-The $Q_{(\text {extr })}$ is the extracts HRR calculated under the smoke vent.

-The $Q_{(r e s)}$ is the residual HRR calculated in the downstream direction of the duct location.

These quantities can be calculated, using Eq. (3) which can be written as follows:

$Q=\iint U\left[T-T_{0}\right] d \sigma=\sum_{j k} U_{j k}\left[T_{j k}-T_{0}\right] \Delta Y \Delta Z$

Where $U_{j k}$ and $T_{j k}$ are the longitudinal component velocity and the Temperature respectively calculated in each plane, $T_{0}$ is the ambient temperature and $\sigma$ is the cross-section area for each considered plane.

For each simulated case, we kept the same measurement point to calculate the $Q_{(-)}(1 \mathrm{~m})$, and 
the $Q_{(+)}(3.7 \mathrm{~m})$, however, the $Q_{(\text {extr })}$ and the $Q_{(r e s)}$ will be changed from one case to another.

So, for the three sets of numerical simulations, the duct efficiency is determined depending on the $Q_{(e x t r)}$ and is calculated using the Eq. (2).

\section{RESULTS AND DISCUSSION}

\subsection{Efficiency and Temperature Distribution}

In this sub-section, first the efficiency of the duct system is calculated, and then the temperature distributions in the tunnel and in the duct are examined, while considering both the effect of the duct section, height and shape.

\subsubsection{Influence of the duct section}

Figure 3 shows the efficiency of the duct for different cross-sections [0.01-0.36 $\left.\mathrm{m}^{2}\right]$. It can be seen from this figure that the efficiency increases with the increase of the duct section.

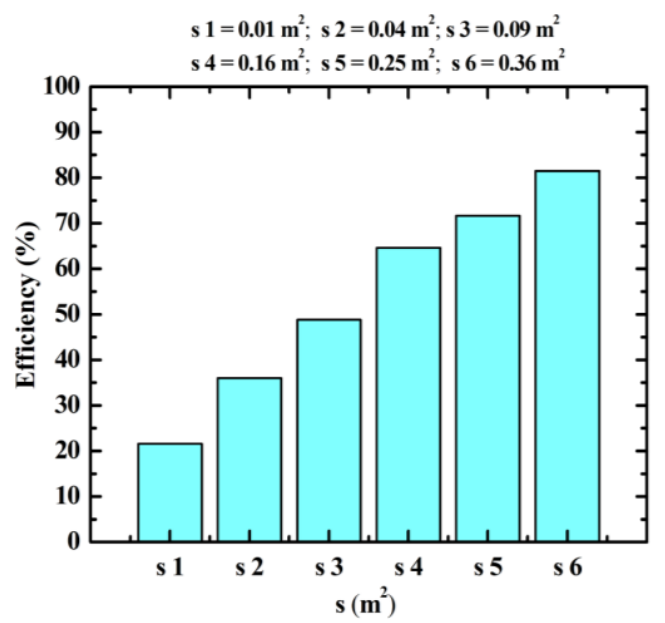

Fig. 3. Duct efficiency for different duct section.

It can be noticed that the minimum efficiency is obtained for the smaller duct $\left(0.01 \mathrm{~m}^{2}\right)$, with $21.57 \%$ and the maximum efficiency is realized in case of the $0.36-\mathrm{m}^{2}$ duct, with $81.46 \%$. So, the 100 $\%$ efficiency is far from being reached. It is crystal clear that there is an extended mixing process in the tunnel center (Partial enhancement of smoke layer) into the tunnel ceiling, and a low quantity of air that has already entered the bottom of the large duct $(L=l=0.6 \mathrm{~m})$ through the leakage area. This can be explained by the fact that when the horizontal velocity of the smoke flowing into the duct becomes higher, the vertical inertia force induced by stack effect becomes quite weaker where the smoke flow is controlled by the horizontal inertia force.

As a result, a small quantity of fresh air can only be discharged from the duct and the effective smoke exhausting area can slightly decrease. Consequently, we obtain about $4 / 5$ of the total efficiency of the smoke exhausting in the bigger duct.

To quantify the influence of the mixing process on the natural exhausting effect more accurately, the representative selections of lateral temperature contour in the tunnel and the duct for different duct sections are illustrated in Fig. 4 (where the left side reveals the isotherm distribution in the tunnel). Results for duct selections of $0.01 \mathrm{~m}^{2}, 0.09 \mathrm{~m}^{2}, 0.25$ $\mathrm{m}^{2}$ and $0.36 \mathrm{~m}^{2}$ are selected here.

As shown in Fig. 4(a), when the disturbance on the smoke layer interface starts to break the stability of the smoke under the small effect of the vertical inertia force, a small quantity of fresh air mixed into the smoke layer and a very small sunken area appears. The highest point of the sunken area is still smaller than the lowest point opening of the ceiling. The smoke in the upper layer is unable to separate from this region and then to exhaust through the duct section of $0.01 \mathrm{~m}^{2}$. So, this is the cause of the smoke exhausting inefficiency for this case as it is shown in Fig. 3.

However, in cases with a duct section larger than $0.01 \mathrm{~m}^{2}$, and under the average driving effect of the vertical inertia force, it is very clear that the highest points of the ceiling sunken areas appear more clearly (Figs. 4(b), 4(c) and 4(d)). The lower limit layer starts to interact with the upper central area of the tunnel where the flow speed increases rapidly with the duct section increasing and may form a particular gas mixture. This combination of a smoke-air mixture flows across the flight zone and the smoke layer thickness below the duct weakness. So, the highest point enters into the middle of the duct and the mixed fresh air with smoke is drawn into the duct bottom. For the duct section of 0.09 $\mathrm{m}^{2}$, the smoke temperature in the middle region of the lower part of the duct is about $23^{\circ} \mathrm{C}$ and there exists an area where the thermal field reaches the $29^{\circ} \mathrm{C}$ value. It is very clear that the proportion of the mixed air is exhausted with the smoke through this average section and will be the cause of the smoke exhausting inefficiency (Fig. 3 and Fig. 4(b)). As shown in Figs. 4(c) and 4(d), the temperature at the ceiling level is almost equal to that at the duct bottom. For example, we can notice that the temperature reaches $61^{\circ} \mathrm{C}$ and $39^{\circ} \mathrm{C}$ in the left and in the right parts of the sunken area successively (case for $s=0.36 \mathrm{~m}^{2}$ ). The lower part of the duct is also characterized by a similarity from the thermal field $\left(61^{\circ} \mathrm{C}\right.$ in the left and $39^{\circ} \mathrm{C}$ in the right sides). It is worthwhile to note that a thicker smoke layer with relatively high horizontal inertia force forms will lead to a stable smoke layer under the duct, which restrains the mixed air entrainment in the smoke layer from the left and the right direction and avoids the occurrence of the stack effect. However, under the action of the weak vertical disturbance, the mixture process becomes a bit stronger due to the smoke exhausting of the duct, and a portion of fresh air will be flowing into the center of the duct cross-section. The amount of air which is mixed with the smoke is exhausted by it and the other part 

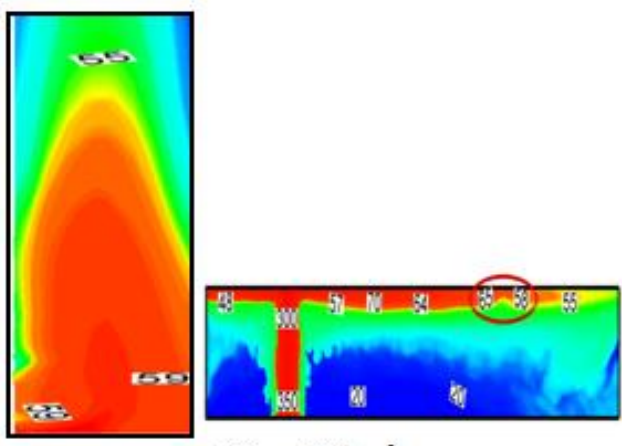

(a) $\mathrm{s}=0.01 \mathrm{~m}^{2}$

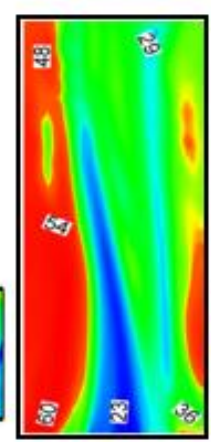

(b) $\mathrm{s}=0.09 \mathrm{~m}^{2}$

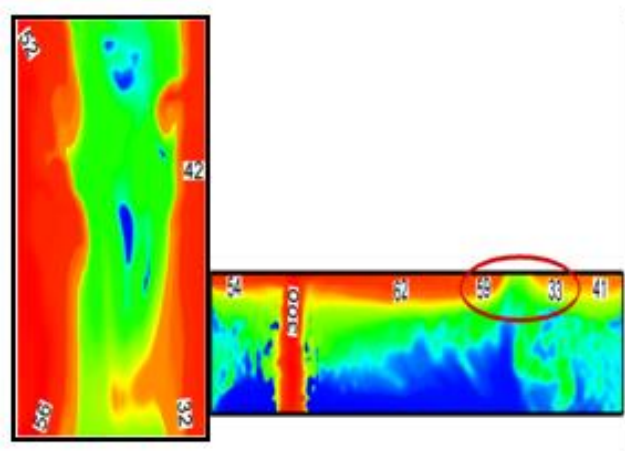

(c) $\mathrm{s}=0.25 \mathrm{~m}^{2}$
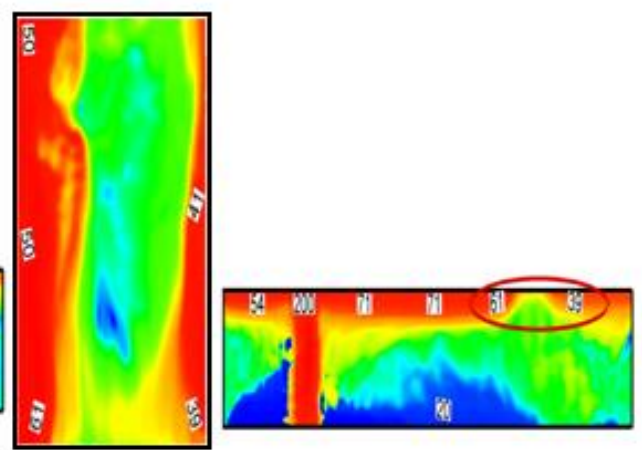

(d) $\mathrm{s}=0.36 \mathrm{~m}^{2}$

Fig. 4. Temperature Contour distribution with various duct section in the corridor (The zone sunken is circulated on red) and in the duct (In the left side: duct system; In the right side: the corridor).

remains in the bolt position between the thicker smoke layers in the medium. The rest keeps spreading along the tunnel ceiling. Therefore, the smoke efficiency is relatively large with the largest section, as presented in Fig. 3.

\subsubsection{Influence of the duct height}

Figure 5 shows the duct efficiency for different duct heights. As shown in this figure, the efficiency is slightly increased when the duct height increases.

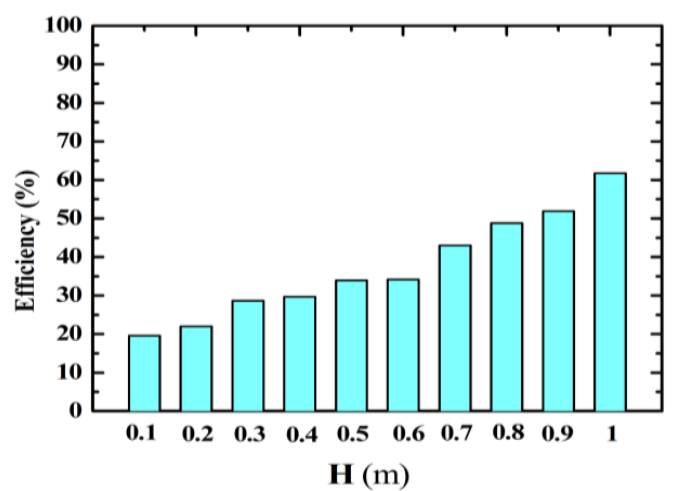

Fig. 5. Duct efficiency for different duct height.

At duct height below $0.7 \mathrm{~m}$, the increasing from one case to another is so neglected and the efficiency does not exceed the $35 \%$, indicating that the exhaust smoke efficiency based on height vertical duct below $0.7 \mathrm{~m}$ is not significant. At $0.7 \mathrm{~m}$ height, the realized efficiency is well expected (42.99\%) as it is mentioned later. When the duct reaches the height of $1 \mathrm{~m}$, we obtained the maximum percentage $(61.75 \%)$ and the efficiency seems to be with an average value where the duct height becomes comparable to the tunnel height size. It can be seen that the flow of the smoke motion is dominated by the vertical inertia force induced by a stack effect and this force of smoke increases as the duct height increases. The disturbance on the smoke layer interface will be greatly strengthened, leading to a lot of more fresh air drawn in the smoke layer and the flowing smoke to the duct weakens progressively. The reason is that when the vertical inertia force is large, the fresh air beneath smoke layer is directly exhausted and plug-holing occurs, resulting in the duct efficiency reduction and the exhausting process of the smoke flow is controlled by the vertical inertia force of stack effect.

To illustrate the plug-holing phenomena appearance more exactly and to compare the effect of different duct heights on natural smoke exhausting efficiency, we propose to study the temperature distribution in the duct and the tunnel with several duct heights under $s=0.09 \mathrm{~m}^{2}$ (Fig. 6). Results for duct heights of $0.5 \mathrm{~m}, 0.7 \mathrm{~m}, 0.9 \mathrm{~m}$ and $1 \mathrm{~m}$ are selected here.

As it is shown in Figs. 6(a) and 6(b), the sunken area and the height of the highest point gradually increase with the duct height increase. When the height is $0.5 \mathrm{~m}$, the highest point reaches the duct medium under a weak vertical current of a flue air where the temperature is about $24^{\circ} \mathrm{C}$ in the base of the air stream and about $29^{\circ} \mathrm{C}$ at the top. 

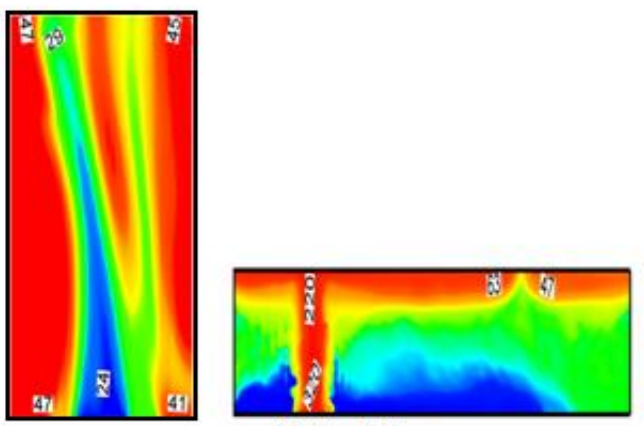

(a) $\mathrm{H}=0.5 \mathrm{~m}$
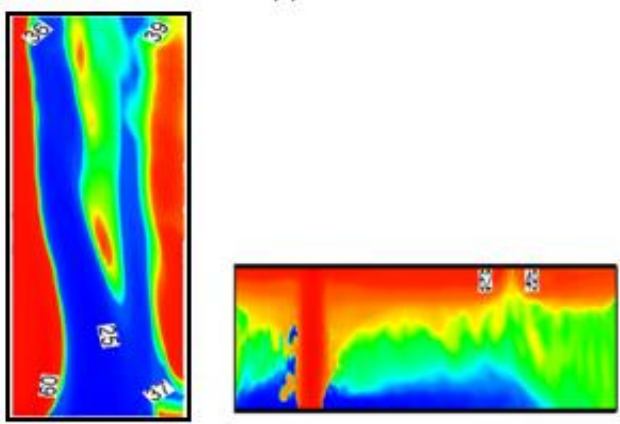

(c) $\mathrm{H}=0.9 \mathrm{~m}$

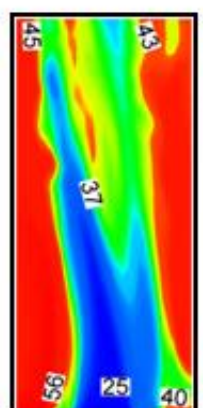

40
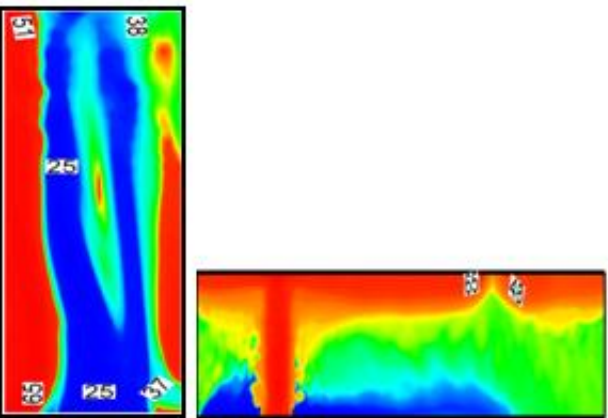

(d) $\mathrm{H}=\operatorname{lm}$

Fig. 6. Temperature contour distribution with various duct height in the corridor and in the duct (In the left side: duct system; In the right side: the corridor).

For $h=0.7 \mathrm{~m}$, the lower limit of the smoke layer starts to interact with the center limit area of the tunnel application. The highest point appearance ameliorates and induces the beginning of a secondary vertical stream of flue-air emergence, where the primary flue stream will expand gradually without flowing-up the duct top. Hence, the hot smoke is exhausted upstream and downstream as an adhered spill plume from the duct. With the duct height increase, Figs. 6(c) and 6(d) show that the smoke layer beneath the vent under the tunnel ceiling shows a remarkable sunken area. As the disturbances on the smoke layer interface are strong, the intensity of the stack effect induced by the hot smoke flowing into the duct from the tunnel is gradually enhanced. Simultaneously, the smoke layer under the ducttunnel contact is thinner. For these reasons, the strong vertical inertial force of the stack effect will easily break the stratification of the smoke layer in this region. When the smoke layer thickness beneath the vent decreases to 0 , the apex of the depression enters the duct and the highest point reaches the duct top. Then, a lot of air will be exhausted directly by the duct. This special phenomenon in the smoke exhaust process is called plug-holing, i.e. the fresh air is drawn directly into the duct from the lower layer. Obviously, the area filled with cold air increases with the height increase. It is obvious that Fig. 6(c) indicates the presence of two currents of flue cold-air which spread along the duct top and promote the weakness of the adhered spill plumes. The temperature distribution is about $25^{\circ} \mathrm{C}$ in the duct bottom and about $36^{\circ} \mathrm{C}$ in the top. In Fig. $6(\mathrm{~d})$, the primary and the secondary inner sections of the flue cold-air stream start to interact with each other at the upper part of the duct where the downstream adhered spill plume weakens more once again. Therefore, a lot of air is easily exhausted by the duct.

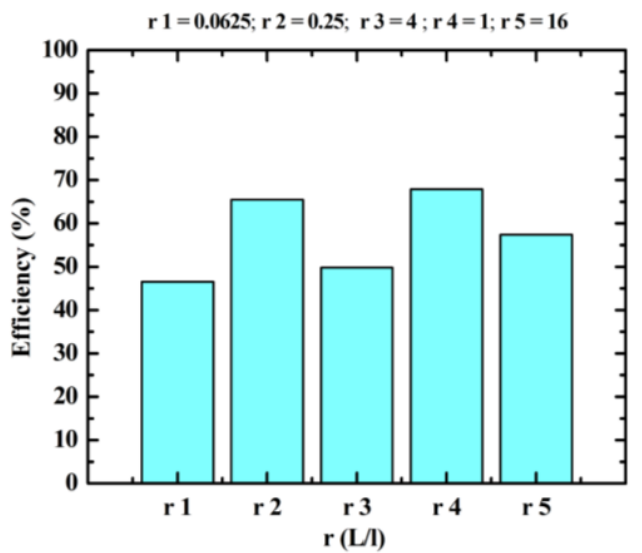

Fig. 7. Duct efficiency as a function of different aspect ratio (r): Variation of the duct shape.

\subsubsection{Influence of the Duct Shape}

In this section, five different shapes are tested by changing the ideal case orientation $(0.32,0.32)$ by raising or reducing the length and the width of the duct separately, as it is presented in part (2.1). Figure 7 shows the presence of two principal levels of the peaks-efficiency. The first level is characterized by the lower peaks, which correspond to the $r=0.0625$ and $r=1$. In this case, even $50 \%$ is not yet reached, resulting in poor natural ventilation performance. The second, is 

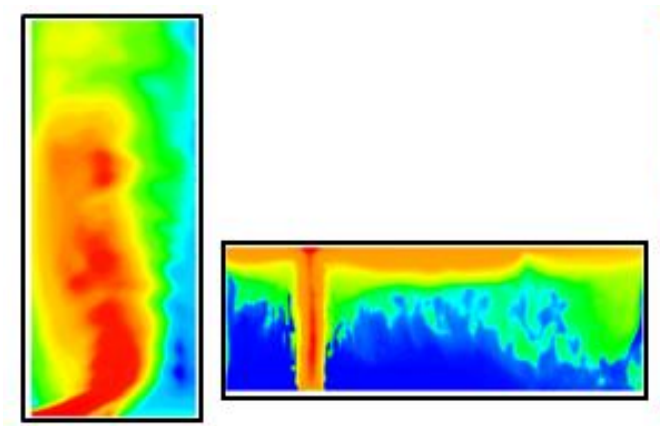

(a) $\mathrm{r}=0.25$
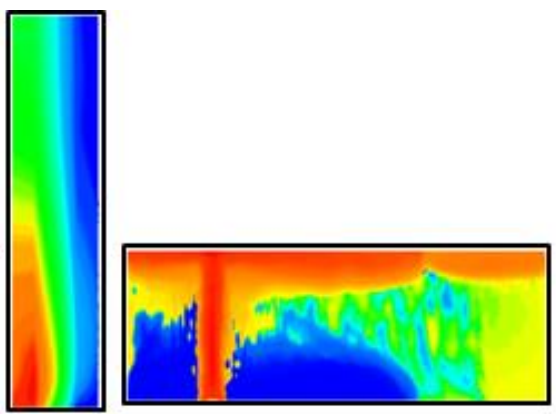

(b) $r=0.0625$

Fig. 8 Temperature contour distribution with various duct shape in the corridor and in the duct.

characterized by the higher peaks which correspond to the $r=0.25,4$ and 16 . It is very clear that the efficiency does not exceed an average value for the smoke extraction $(67.88 \%)$ and especially for different aspect-ratio, which would be expected. Indeed, for the first numerical simulation and for the cross-section of the duct with $0.16 \mathrm{~m}^{2}$, the efficiency is about $64.59 \%$. So, we can conclude that on the one hand the efficiency of the smoke extraction is not affected by the duct shape variation with a $0.16 \mathrm{~m}^{2}$ of cross-section. On the other hand, it is quite interesting to note that despite the greatest value of the aspect-ratio (4 and 16) and according with this stability of the cross-section, the efficiency always maintains a relatively steady value at about $46-67 \%$.

Considering this parameter comprehensively, it can be concluded that the duct shape effect is insignificant. Therefore, we are interested here in examining the temperature contours for two different levels of efficiency. As it is illustrated in Fig. 8, the temperature distributions in the duct and tunnel for $r=0.25$ and 0.0625 are presented. Figure 8(a) shows that, after penetration, the hot smoke is exhausted upstream, as an adhered spill plume, from the duct. It can be seen that there exists an area in the tunnel center indicating that the mixing process is well established between the lower part of the smoke layer and the cold air. When the highest point just reaches the duct bottom, the cold air flows through the right side under a vertical flue stream which weakens in the duct top and causes the smoke exhausting effect reduction. So, this is the cause of the efficiency decrease at about $65.44 \%$ resulting in average natural ventilation effectiveness. Figure 8(b) reveals that the mixing process in the tunnel increases in case of $r=0.0625$. Obviously, a part of the smoke layer descends near the ground and the area filled with cold air increases. As shown in Fig. 8(b), the cold air has been completely flowing through the vertical flue stream under the duct downstream, indicating that the plug-holing is mainly produced. Then, the 2D flow pattern weakens and occupies just the upstream direction of the duct medium. It presents here the inefficiency process of the hot smoke exhausting by the duct upstream. So, the effective smoke exhausting area reduces significantly $(46.52$ $\%$ ), resulting in a poor natural ventilation performance.

\subsection{Thermal Stratification Characteristics: Influence of the Newman Parameter " $S$ "}

During a fire in a tunnel, the stratification stability represents the essential evacuation condition to the traffic congestion. Since the buoyancy force pushes in the upper part of the tunnel the hot and the light particles, the denser particles stay on the ground. Thus, along the downstream direction of the plume, a light layer overcomes the heavier layer. This is the case of a stable stratification. However, in the presence of natural exhaust duct in the roofopening, different phenomena can produce the stratification instability (as it is shown in part 4.1.1 and 4.1.2).

In Newman's study, a quantitative criterion was examined. It is defined as follows:

$S=\frac{T_{c}-T_{f}}{T_{a v r g}-T_{0}}$

Let $T_{c}$ is the temperature of fluid in the vicinity of the tunnel ceiling, $T_{f}$ is the temperature in the vicinity of the ground, $T_{\text {avrg }}$ is the average temperature and $T_{0}$ is the ambient temperature. Newman claimed that a sufficient stratification is characterized by a significant difference between the temperature on the floor and at the ceiling, and thus an average temperature is not relatively higher than the initial temperature.

Newman defines two zones depending on the critical value. Thus the $S>1.7$ condition indicates that the temperature at the ground is equal to the initial temperature and the temperature difference between the floor and the ceiling is relatively large. So, the preserved condition for $S>1.7$ seems a good criterion to judge the smoke stratification and based on the smoke flow patch direction, the $S$ parameter evolution can reflect the smoke layer situation under several scenarios.

Newman (1984) found that best results are obtained from $X / h=2$, to avoid any perturbation of the heat plume. In our study, this condition corresponds to $X / h=3.13$. However, we have extrapolated measurement at a distance $X / h=3.5$ to give more accuracy of the downstream layer coexistence. 


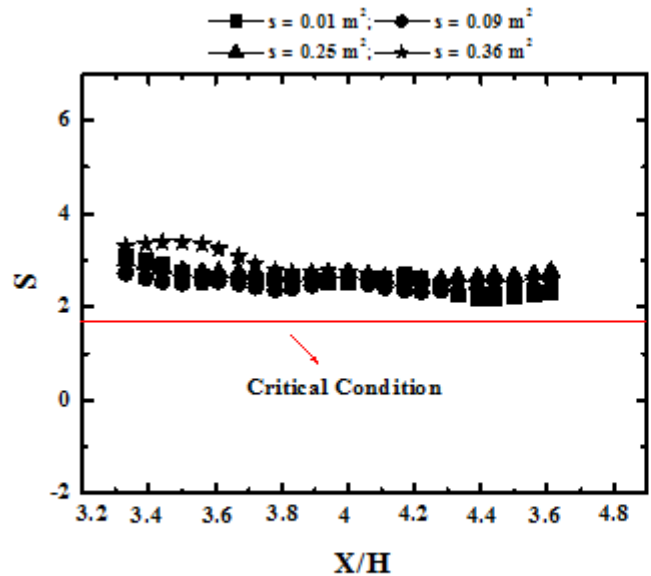

(a)

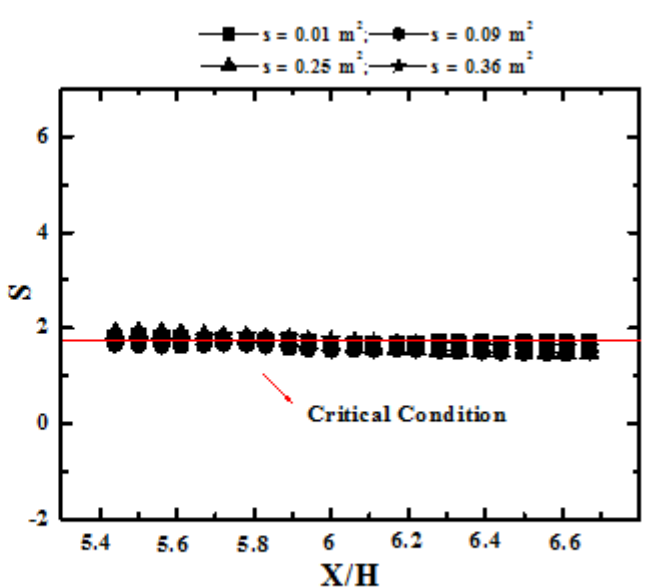

(b)

Fig. 9. The longitudinal evaluation of the stratification parameter (based on Newman, 1984) with the duct section variation: (a) the upstream view, (b) the downstream view.

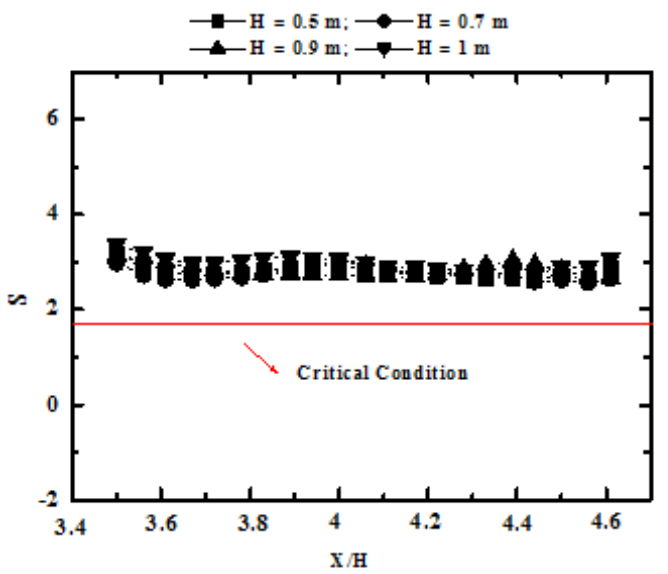

(a)

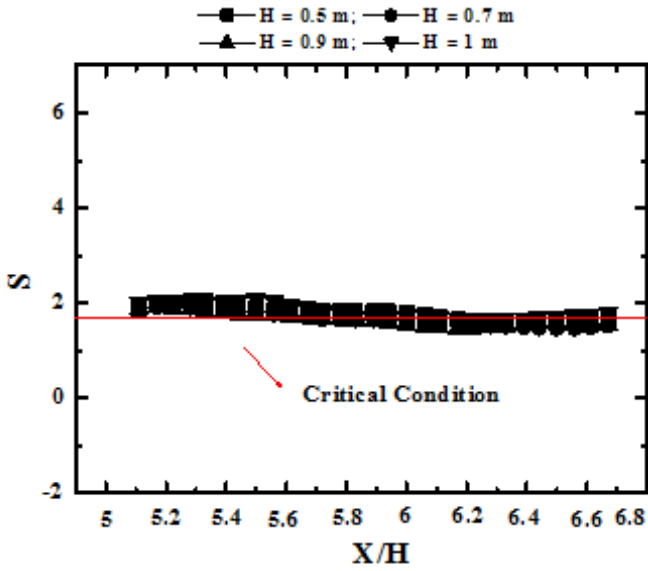

(b)

Fig. 10. The longitudinal evaluation of the stratification parameter (based on Newman, 1984) with the duct height variation: (a) the upstream view, (b) the downstream view.

Figures 9 to 11 present the longitudinal stratification parameter profiles as a function of the dimensionless length in the tunnel $(X / h$, where $h$ is the tunnel height) and successively for different duct sections, duct heights and duct shapes. It is calculated in the downstream direction and in the plane $(Y=1)$.In the upstream duct direction (Figs. 9(a), 10(a) and 11(a)), the stratification parameter is spatially uniform and seems to be higher than the critical condition with the duct geometric parameter variation. The smoke layer is stabilized, and the steady-state will stand in the $S$ range of [2-3.5]. In the downstream duct direction (Figs. 9(b), 10(b) and 11(b)), visualizations show that the stratification parameter decreases and takes a decreasingly 2D line shape for all the data. It is very clear that for different duct geometry variation, the longitudinal $S_{N}$ parameters produce a significant effect on the exit upstream flow and do influence the initial superficial layer intensities. When the smoke flows across the right trailing edge of the duct, the impact between the two systems occurs immediately and the flow field structure changes, resulting in flow resistance increasing. This impact implies a significant rate of air entrainment in the interface zone and more air is entrained into the smoke layer. The temperature gradient decreases progressively since more dilution will be required to make the entrained fluid ambient. So, the stratification parameter reduces progressively, and the stratification stability will be broken.

Based on these ascertainments, we plotted the longitudinal distribution of the stratification parameter in the downstream direction of the duct as it is shown in Figs. 9(b), 10(b) and 11(b).

With the duct section variation, Figure 9(b) indicates the presence of two principal regions which depend on the steady-state emergence of the smoke layer stratification. The first one is situated above the straight line of the critical condition $(S>1.7)$ and indicates the stability existence of the stratification. However, the second one is situated below $(S<1.7)$ the line of the steady-state, 


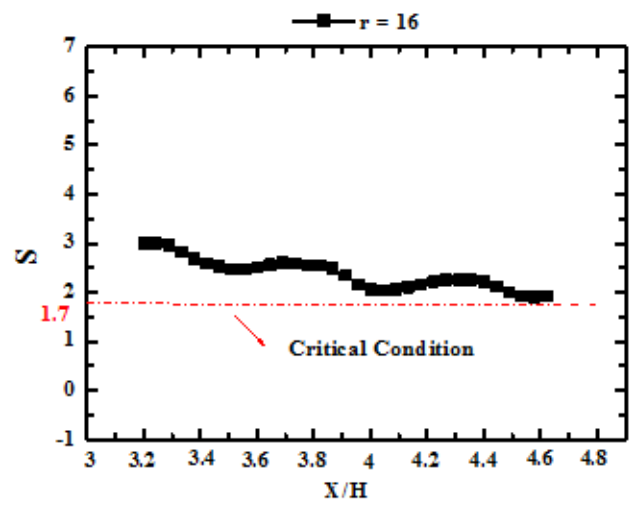

(a)

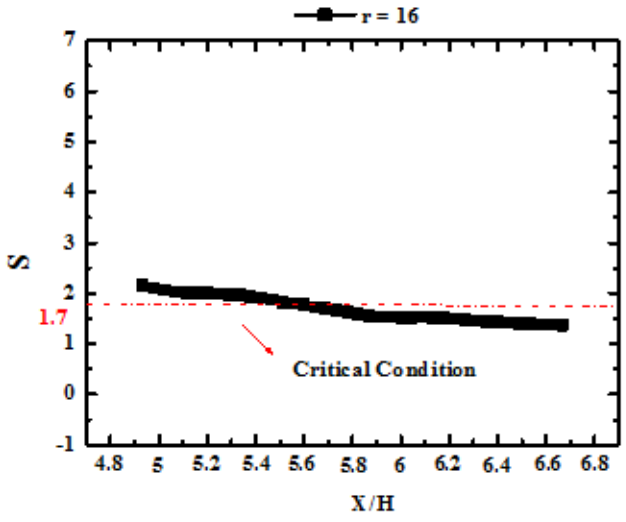

(b)

Fig. 11. The longitudinal evaluation of the stratification parameter (based on Newman, 1984) for $r=$ 16: (a) the upstream view, (b) the downstream view.

indicating that the downstream layer localization to the duct system is well detected.

Figure 10(b) reveals the stratification parameter evolution for different duct heights along the dimensionless length $(X / h)$ and in the right side of the duct. It can be seen that there is a strong influence on the turbulent entrainment process and the $\mathrm{S}$ basically behaves in the non-stability state (presence of points below the critical condition), where the cold layer collapses in the inner surface and produces the broken downstream of the hot layer.

These analyses are not surprising and had previously been found in a good agreement with the behavior illustrated state obtained in part (4.1.2).

Figure 11(b) presents the stratification parameter distribution at a given aspect ratio $(r=16)$. It can be seen from this figure the perturbation that is detected on the downstream layer from this shape of the duct. We can estimate the emergence of two different zones:

$4.8 \leq X / h \leq 5.4$, the stratification reaches the stability state $(S>1.7)$.

$X / h \geq 5.7$, the stratification is broken $(S<1.7)$.

We can conclude that at a given downstream satisfactory emplacement at about $X / h=3.5$, the stratification pattern can be described by the $2 \mathrm{D}$ flow pattern. Despite the similarity that was observed for all the presented tests and for different duct dimensional characteristics, we note that the buoyant flow stratification at the upstream direction from the duct location was stable. However, the stratification pattern in the downstream direction remains unstable and the degree of the instability depends of the duct geometric parameters. So, it depends on the data distribution of $S$.

\section{CONCLUSIONS}

Numerical simulations were conducted to investigate the smoke extraction efficiency together with the thermal stratification of the downstream smoke layer. Different models of a ceiling geometric-duct variation were performed in a corridor fire with Large Eddy simulation (LES).

Analysis of these results has provided the following conclusions.

- With the fixed duct section, it appears that when the duct height is relatively small, the smoke exhausting process is inefficient. With the duct height increasing, the plug-holing phenomenon is well established and is controlled by the vertical inertial force. In this case, the flow pattern is immediately determined with the combined function of the assumption of 2D flow pattern in near region and the two vertical streams of ambient flue air. These two observations are applicable to predict the process of hot smoke exhausted by the duct upstream and downstream after penetration.

- It is suggested that the section of the duct appears as the most influent dimensional parameter and the efficiency increases with the duct section increase. In cases of the higher duct crosssections, the hot smoke is discharged as an adhered spill plume and we obtained the best performance for natural ventilation. However, the $100 \%$ efficiency is not achieved because of the mixing gas discharging throw the larger duct.

- The shape of the duct seems to have no significant influence on the natural extraction efficiency.

- The thermal stratification and the consequent flow patterns correlated well with the Newman stratification parameter. Generally, we found that the stratification parameters are uniform along the longitudinal direction where the " $S$ " parameter profiles are typical of a homogeneous channel flow and the stratification is permitted $(S>1.7)$ in the upstream duct direction. However, the flow seems to be unstable in the downstream duct direction $(S<1.7)$ and the unstability degree 
depends on the duct-system geometry.

\section{REFERENCES}

Alarie, Y. (2002). Toxicity of fire smoke. Critical Reviews in Toxicology 32, 259-289.

Carvel, R.O. and G. Marlair (2005). A history of experimental tunnel fires. Beard AN, Carvel RO, editors, The handbook of tunnel fire safety, London 199 -230.

Gann, R.J., V. Babrauskas and R. D. Peacock. (1994). Fire conditions for smoke toxicity measurements. Fire and Materials 18(3), 193199.

Hong, W. H. (2004). The progress and controlling situation of Daegu Subway fire disaster. In Proceedings of the sixth Asia-Oceania symposium on fire science and technology, Korea 28- 46.

Hwang, C. C. and J. C. Edwards (2005). The critical ventilation velocity in tunnel fires-a computer simulation. Fire Safety Journal 40, 213-244.

Ji, J., J. Y. Han, C. G. Fan, Z. H. Gao and J. H. Sun (2013). Influence of cross-sectional area and aspect ratio of shaft on natural ventilation in urban road tunnel. International Journal of Heat and Mass Transfer 67, 420-431.

Ji, J., L. Kaiyuanb, Z. Wei and H. Ran (2010). Experimental investigation on influence of smoke venting velocity and vent height on mechanical smoke exhaust efficiency. Journal of Hazardous Materials 177, 209-215.

Ji, J., Z. H. Gao, C. G. Fan, W. Zhong and J. H. Sun (2012). A study of the effect of plug-holing and boundary layer separation on natural ventilation with vertical shaft in urban road
Tunnel fires. International Journal of Heat and Mass Transfer 55, 6032-6041.

McGrattan, K., S. Hostikka, J. Floyd, H. Baum, R. Rehm, W. Mell, and R.M. Dermott (2010). Fire Dynamics Simulator (version 5)Technical Reference Guide, National Institute of Standards and Technology, Baltimore, Maryland, NIST Special Publication 1018-5.

McGrattan, K., R.M. Dermott, S. Hostikka, and J. Floyd (2010). Fire Dynamics Simulator (version 5)-User's Guide. National Institute of Standards and Technology, Baltimore, Maryland, NIST Special Publication 1019-5.

Mos, A. (2005). Phenomenological models for fire simulation in road tunnels. Ph. D Thesis, University Claude Bernard, Lyon, France.

Newman, J. S. (1984). Experimental Evaluation of Fire-Induced Stratification. Combustion and Flame 57, 33-39.

Smagorinsky, J. (1963). General circulation experiment with the primitive equations, I. The basic experiment. Monthly Weather Revifew 9, 99-164.

Vauquelin, O. (2008). Experimental Simulations of fire-induced smoke control in tunnels using an air-helium reduced scale model: Principle, limitation, results and future. Tunnelling and Underground Space Technology 23, 171-178.

Vauquelin, O. and O. Mégret (2002). Smoke extraction experiments in case of fire in a tunnel. Fire Safety Journal 37, 525-533.

Zhong, W., C. G. Fan, J. Ji and J. P. Yang (2013). Influence of longitudinal wind on natural ventilation with vertical shaft in a road tunnel fire. International Journal of Heat and Mass Transfer 57, 671-678. 